\title{
MORBIDLY ADHERENT PLACENTA IN A PRIMI GRAVIDA FOLLOWING INCOMPLETE ABORTION: A CASE REPORT
}

\section{S. R. Mudanur ${ }^{1}$, Shama Mallesh ${ }^{2}$, Neha Chandra ${ }^{3}$}

\section{HOW TO CITE THIS ARTICLE:}

S. R. Mudanur, Shama Mallesh, Neha Chandra. "Morbidly Adherent Placenta in a Primi Gravida following Incomplete Abortion: A Case Report". Journal of Evolution of Medical and Dental Sciences 2015; Vol. 4, Issue 27, April 02; Page: 4742-4746, DOI: 10.14260/jemds/2015/688

ABSTRACT: With rising incidence of caesarean sections and the number of cases of placenta praevia, incidence of morbidly adherent placenta is on the rise. Morbidly adherent placenta, which includes placenta accreta, Increta, and percreta, implies an abnormal implantation of the placenta into the uterine wall. Sonographic markers of placenta accreta can be present as early as the first trimester, such as low uterine implantation of a gestational sac, multiple vascular lacunae within the placenta, loss of the normal hypoechoic retroplacental zone and abnormality of the uterine serosa-bladder interface. Ultrasound has high sensitivity and specificity for the diagnosis of placenta accreta. MRI should be reserved for rare cases in which ultrasound is non-diagnostic. The successful management of placenta accreta includes a team approach with the successful management relying on the prenatal diagnosis of this entity and preparing for the surgical management. Antenatal diagnosis and management in a tertiary care centre helps to reduce maternal and neonatal morbidity and mortality. ${ }^{1}$ We present a patient in whom the antenatal diagnosis of morbidly adherent placenta was missed due to lack of antenatal care and presented as second trimester spontaneous incomplete abortion.

KEYWORDS: Accreta; Increta; Morbidly adherent placenta; Percreta; Peripartum hysterectomy;

INTRODUCTION: Morbidly adherent placenta (MAP) is a rare complication of human placentation that may threaten maternal life due to massive haemorrhage. MAP occurs when there is a defect in the decidua basalis, resulting in abnormal invasion of the trophoblast into the myometrium. Normally the placenta adheres to decidua basalis layer, allowing for a smooth separation of the placenta from the uterus after delivery. In MAP there is no clear plane of cleavage between the placenta and the underlying myometrium. Depending on the extent of adherence and invasion of the placenta the condition is classified as placenta accreta (reaching the myometrium), placenta increta (invading into the myometrium) and placenta percreta (penetrating through the myometrium to breach the serosa or beyond). It is thought to be due to an absence or deficiency of Nitabuch's layer or the spongiosus layer of the decidua. It happens as a consequence of failure of reconstitution of the decidua basalis after repair of caesarean incision, thus resulting in absence of intervening decidual tissue between the invading trophoblast and the myometrium. It has been proposed that the abnormality of the placental uterine interface leads to leakage of fetal alpha-fetoproteins into the maternal circulation resulting in elevated levels of maternal serum alpha fetoproteins. ${ }^{1}$

Major risk factor for MAP is Placenta previa in a woman with previous Caesarean section. Women with a previous cesarean section (CS) require a high index of suspicion as there are two problems to exclude: placenta previa and placenta accreta. Placenta previa itself raises the risk for accreta due to implantation over a highly vascular, poorly contractile lower uterine segment and an existing scar in the same area compounds the risk. Other risk factors include previous uterine 


\section{CASE REPORT}

surgery, previous dilatation and curettage, previous myomectomy, Asherman syndrome (endometrial defects), submucous leiomyomata, advanced maternal age, multiparity and tobacco use. ${ }^{2}$

CASE REPORT: A young 20 years old, nulliparous lady presented with bleeding per vagina, fever and generalised weakness. She had undergone Dilatation \& Curettage for second trimester spontaneous incomplete abortion 5 months back. Post abortal period she had spotting daily for a month. She was found to have retained products of conception on USG and underwent check curettage again. She presented 3 months later with the above complaints. On pelvic examination cervix and vagina was found healthy with minimal white discharge with bulky and retroverted uterus. Urine pregnancy test was positive and the serum beta hCG on the day of admission was $763.72 \mathrm{mIU} / \mathrm{ml}$. On USG uterus was found enlarged and bulky measuring $102 * 71 * 58 \mathrm{~mm}$, a hyperechoic lesion was found in the uterine cavity measuring $68 * 34 \mathrm{~mm}$ suggestive of retained placenta. She again underwent check curettage and profound placental tissue was extracted with associated excessive bleeding. She was transfused with 1 unit of packed cells and she received parenteral iron therapy (iron sucrose). Post-operative period, she was found to have raised antibody titres for enteric fever (widal test) and treated with cephalosporins (ceftriaxone). Her subsequent UPT performed 3 days after check curettage was negative and serum hCG level had reduced to $2.3 \mathrm{mIU} / \mathrm{ml}$. She recovered from her symptoms and was discharged after a check scan which showed no retained products.

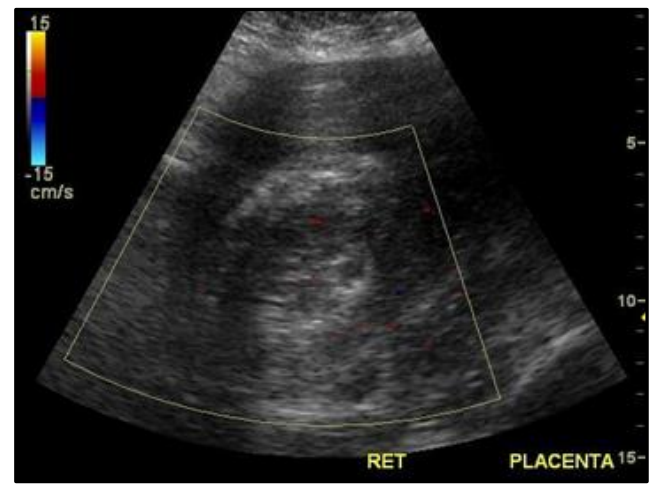

\section{Fig. 1: USG image shows retained placenta}

DISCUSSION: Placenta accreta is the most common reason for emergency postpartum hysterectomy and worldwide mortality rate is $7 \%-10 \% .^{3}$ Clinical consequences of placenta accreta are uterine perforation, massive haemorrhage at the time of placental separation, infection, hysterectomy and associated co-morbidites such as ureteral injury and fistula formation (5\%), need for transfusion (90\%) and mortality (7.4\%).4 Antenatal diagnosis is the single most important factor in improving the outcome in MAP.

The risk of placenta accreta increases from 3.3\% in patients with history of one caesarean section and placenta previa to $11 \%$ in patients with history of two caesarean section and placenta previa and the risk rises to $40 \%$ with history of three caesarean section and placenta previa. While without placenta previa the risk of placenta accreta is $0.03 \%$ in patients with history of one caesarean section, $0.1 \%$ in patients with history of two cesarean sections and $0.2 \%$ with history of three caesarean sections. ${ }^{5}$ 
Ultrasonography is the first-line investigation for suspected placental invasion of the myometrium. The two diagnostic criteria used are presence of irregular lacunae within the placenta and the loss of retroplacental clear space. Irregularly shaped placental lacunae within the placenta and turbulent flow through these lacunae during Doppler flow studies have a diagnostic sensitivity of $79 \%$ at 15 to 20 weeks of gestation and $93 \%$ after 20 weeks. These lacunae may give the placenta a "moth-eaten" or "swiss cheese" appearance. The risk of MAP increases with the rise in the number of lacunae. The second diagnostic feature is loss of retroplacental "clear space". Other less-sensitive criteria include thinning or absence of the myometrium overlying the placenta, increased vascularity of the uterine serosa-bladder interface and protrusion of the placenta into the bladder.

DOPPLER STUDY: There is usually an increase in vascularisation of the placental-myometrial interface in MAP. Magnetic resonance imaging (MRI) has been effectively used for the investigation of placental invasion. There are three significant MRI features for the detection of MAP. These are: abnormal uterine bulging of the normal pear-shaped gravid uterus, heterogeneity of the signal intensity of the placenta on T2-weighted images and the presence of T2- weighted dark linear bands in intraplacental signal intensity, extending from the basilar plate to the placental surface. Cystoscopy is useful in cases of placenta percreta where involvement of the bladder is suspected, but biopsy should be avoided as it may precipitate severe haemorrhage. Placing ureteral stents during cystoscopy helps in intra-operative identification of the ureters. ${ }^{1}$

TREATMENT: A team approach is relevant in managing these patients in order to reduce morbidity and mortality associated with MAP. Consideration should be given to anticipation and management of massive haemorrhage. Blood components, including packed cells, platelets, fresh frozen plasma, cryoprecipitate, and activated factor VII should be arranged. Antenatal diagnosis enables one to leave the placenta undisturbed, either during caesarean hysterectomy or in managing the MAP conservatively. Pre-operative and/or intra-operative ultrasound mapping, to delineate the area of the uterus overlying the placenta prior to the uterine incision is useful. Traditional management of MAP is caesarean hysterectomy. Over the last decade, management of MAP has changed from the old obstetric dictum of never leaving any part of the placenta in utero, to a more conservative approach. ${ }^{4}$

Devascularising the pelvis, Balloon Catheter Occlusion of the pelvic vessels or Selective arterial embolisation decreases the blood flow to uterus. Bilateral internal iliac artery ligation is performed prior to peripartum hysterectomy in an attempt to reduce operative blood loss. This is of importance in situations where interventional radiology is not available.

Other surgical options: It is possible to "excise the placental site". If the area of placental attachment is focal and the majority of the placenta has been removed, then a "wedge resection" of the area can be performed.

Conservative approach: In this, once the baby is delivered the placenta is left undisturbed, the cord is cut short and the uterine incision is closed.

Postoperative complication: Include DIC, fistula formation, ureteral stricture, urinary stasis, infection, pelvic and renal abscess formation, Renal compromise, transfusion reaction, sepsis, ARDS, multi organ failure. 
Uterus preserving modalities should be considered only in highly selected cases when blood loss is minimal and there is desire for fertility preservation. The conservative approach of managing MAP was first described by Arulkumaran and reported administration $50 \mathrm{mg}$ of methotrexate as an intravenous infusion on alternate days and the placental mass was expelled during postnatal period 6 . Methotrexate, a folate antagonist acts primarily against rapidly dividing cells hence effective against dividing trophoblast.

Subsequent management of the placenta: The placenta is left to reabsorb or expel spontaneously. If significant bleeding develops a manual removal is attempted. It should be noted that interval removal of the placenta can be associated with heavy bleeding.

Use of serum $\boldsymbol{\beta}$ human chorionic gonadotropin and ultrasonography in monitoring: When the placenta is left in situ the woman requires follow up to ascertain resolution of placental tissue as there is a risk of infection and haemorrhage. Clinical condition of the patient, serial USG and $\beta$ hCG are used in this regard. Serum $\beta$ HCG levels are performed on a weekly basis, until it becomes undetectable.

CONCLUSION: A majority of MAP are diagnosed during the third stage of labour or during caesarean section which results in adverse consequences including exsanguinating haemorrhage. A high degree of clinical suspicion coupled with imaging and other relevant investigations like cystoscopy is important in arriving at an antenatal diagnosis of MAP. Although caesarean hysterectomy was the cornerstone in the management in the past, antenatal diagnosis permits effective and safe conservative approaches today ${ }^{7}$.

\section{REFERENCES:}

1. Herath R P, Wijesinghe P S. Management of morbidly adherent placenta. Sri Lanka Journal of Obstetrics and Gynaecology. 2011; 33: 39-44.

2. Zulfikir S, Al M B, Kakaria A, Gowri V, Saparamandu A, Al L K. Late complication due to placenta increta left in situ and management options. JNMA J Nepal Med Assoc. 2011 Oct-Dec; 51(184): 196-8.

3. Narang L, Chandraharan E. Management of morbidly adherent placenta. Obstetrics, Gynaecology and Reproductive Medicine.2013; 23: 214-220.

4. Wadhwa L et. al. Morbidly Adherent Placenta (MAP): Lessons learnt. Open Journal of Obstetrics and Gynecology.2013; 3: 217-21.

5. Silver R M, Landon M B, Rouse D J, Leveno K J, Spong C Y, et al. Maternal morbidity associated with multiple repeat Cesarean deliveries. Obstetrics \& Gynecology.2006; 107: 1226-32.

6. Arulkumaran S, Ingemasson I, Ratnam SS et. al. Medical treatment of placenta accreta with methotrexate. Acta Obstet Gynecol Scand. 1986; 65: 285-6.

7. Bashir R, Nawaz S, Saleem F. Morbidly adherent placenta and management options. International Journal of Gynecology \& Obstetrics.2012; 119S3: S261-S530. 


\section{CASE REPORT}

\section{AUTHORS:}

1. S. R. Mudanur

2. Shama Mallesh

3. Neha Chandra

\section{PARTICULARS OF CONTRIBUTORS:}

1. Professor, Department of Obstetrics \& Gynaecology, BLDE University's Sri B. M. Patil Medical College, Vijayapura.

2. Junior Resident, Department of Obstetrics \& Gynaecology, BLDE University's Sri B. M. Patil Medical College, Vijayapura.

FINANCIAL OR OTHER

COMPETING INTERESTS: None
3. Consultant, Department of Obstetrics \& Gynaecology, Mudanur Multispecialty Nursing Home, Vijayapura.

\section{NAME ADDRESS EMAIL ID OF THE CORRESPONDING AUTHOR:}

Dr. S. R. Mudanur,

Professor,

Department of Obstetrics \& Gynaecology, BLDE University's Sri B. M. Patil Medical College, Vijayapura-586103, Karnataka, India.

E-mail: drmudanurs@gmail.com

Date of Submission: 01/03/2015.

Date of Peer Review: 02/03/2015.

Date of Acceptance: 20/03/2015.

Date of Publishing: 02/04/2015. 